ANNALES

POLONICI MATHEMATICI

$81.2(2003)$

\title{
Non-existence of some natural operators on connections
}

\author{
by W. M. Mikulski (Kraków)
}

\begin{abstract}
Let $n, r, k$ be natural numbers such that $n \geq k+1$. Non-existence of natural operators $C_{0}^{r} \rightsquigarrow Q\left(\operatorname{reg} T_{k}^{r} \rightarrow K_{k}^{r}\right)$ and $C_{0}^{r} \rightsquigarrow Q\left(\operatorname{reg} T_{k}^{r *} \rightarrow K_{k}^{r *}\right)$ over $n$-manifolds is proved. Some generalizations are obtained.
\end{abstract}

0. Introduction. Let $n, r$ and $k$ be natural numbers such that $n \geq k+1$. In [1], C. Ehresmann constructed functorially the fiber bundle $K_{k}^{r} M=$ $\operatorname{reg} T_{k}^{r} M / L_{k}^{r}$ of contact $(k, r)$-elements over an $n$-dimensional manifold $M$ and obtained the bundle functor $K_{k}^{r}: \mathcal{M} f_{n} \rightarrow \mathcal{F} \mathcal{M}$ from the category $\mathcal{M} f_{n}$ of $n$-dimensional manifolds and their embeddings into the category $\mathcal{F} \mathcal{M}$ of fibered manifolds and their fibered maps. In [5], I. Kolár, P. W. Michor and J. Slovák studied the problem of how a vector field $X$ on $M$ induces a vector field $A(X)$ on $K_{k}^{r} M$ and proved that for sufficiently large $n$ every natural operator $A: T_{\mid \mathcal{M} f_{n}} \rightsquigarrow T K_{k}^{r}$ is a constant multiple of the complete lifting $\mathcal{K}_{k}^{r}$.

In [6], I. Kolár and the author investigated the naturality problem for bundle mappings $B: K_{k}^{r} M \rightarrow K_{k}^{r} M$ and deduced the so called rigidity theorem for $K_{k}^{r}$ saying that the only natural transformation $B: K_{k}^{r} \rightarrow K_{k}^{r}$ over $n$-manifolds is the identity. They also studied the naturality problem for affinors (i.e. tensor fields of type $(1,1)) C: T K_{k}^{r} M \rightarrow T K_{k}^{r} M$ on $K_{k}^{r} M$ and derived that for sufficiently large $n$ every natural affinor $C: T K_{k}^{r} \rightarrow T K_{k}^{r}$ on $K_{k}^{r}$ over $n$-manifolds is a constant multiple of the identity. Moreover they analysed how a 1-form $\omega$ on $M$ can induce a 1-form $D(\omega)$ on $K_{k}^{r} M$ and showed that for sufficiently large $n$ every natural operator $D: T_{\mid \mathcal{M} f_{n}}^{*} \rightsquigarrow$ $T^{*} K_{k}^{r}$ is a constant multiple of the vertical lifting. Some generalizations of the above results can be found in [8].

Similarly to $(k, r)$-elements, C. Ehresmann introduced the fiber bundle $K_{k}^{r *} M=\operatorname{reg} T_{k}^{r *} M / L_{k}^{r}$ of contact $(k, r)$-coelements over an $n$-dimensional manifold $M$. So, we have the bundle functor $K_{k}^{r *}: \mathcal{M} f_{n} \rightarrow \mathcal{F M}$. In [9], we

2000 Mathematics Subject Classification: 58A05, 58A20.

Key words and phrases: bundle functor, natural operator, jet, connection. 
studied for $K_{k}^{r *} M$ the same naturality problems as for $K_{k}^{r} M$ and proved the same results.

In the present paper we continue the investigation of $K_{k}^{r} M$ and $K_{k}^{r *} M$.

In the first part of the paper, we study the problem of whether a torsion free linear $r$-order connection $\lambda: T M \rightarrow J^{r} T M$ on an $n$-manifold $M$ induces a connection $A(\lambda): T\left(\operatorname{reg} T_{k}^{r} M\right) \rightarrow l_{k}^{r}=\operatorname{Lie}\left(L_{k}^{r}\right)$ on the principal fiber bundle reg $T_{k}^{r} M \rightarrow K_{k}^{r} M$ with structure group $L_{k}^{r}$. This problem is reflected in the concept of natural operators $A: C_{0}^{r} \rightsquigarrow Q\left(\operatorname{reg} T_{k}^{r} \rightarrow K_{k}^{r}\right)$ in the sense of [5]. We prove that if $n \geq k+1$, then there are no natural operators $C_{0}^{r} \rightsquigarrow Q\left(\operatorname{reg} T_{k}^{r} \rightarrow K_{k}^{r}\right)$ over $n$-manifolds. We find an assumption on a bundle functor $F: \mathcal{M} f_{n} \rightarrow \mathcal{F} \mathcal{M}$ under which there are no natural operators $F \rightsquigarrow$ $Q\left(\operatorname{reg} T_{k}^{r} \rightarrow K_{k}^{r}\right)$. We give an example of a bundle functor $F: \mathcal{M} f_{n} \rightarrow \mathcal{F} \mathcal{M}$ $(n \geq k+1)$ and a natural operator $A: F \rightsquigarrow Q\left(\operatorname{reg} T_{k}^{r} \rightarrow K_{k}^{r}\right)$.

In the second part we study the problem of whether a torsion free linear $r$-order connection $\lambda: T M \rightarrow J^{r} T M$ on an $n$-manifold $M$ induces a connection $A(\lambda): T\left(\operatorname{reg} T_{k}^{r *} M\right) \rightarrow l_{k}^{r}$ on the principal fiber bundle $\operatorname{reg} T_{k}^{r *} M \rightarrow$ $K_{k}^{r *} M$ with structure group $L_{k}^{r}$. We prove that if $n \geq k+1$ then there are no natural operators $C_{0}^{r} \rightsquigarrow Q\left(\operatorname{reg} T_{k}^{r *} \rightarrow K_{k}^{r *}\right)$ over $n$-manifolds. We find an assumption on a bundle functor $F: \mathcal{M} f_{n} \rightarrow \mathcal{F} \mathcal{M}$ under which there are no natural operators $F \rightsquigarrow Q\left(\operatorname{reg} T_{k}^{r *} \rightarrow K_{k}^{r *}\right)$. We observe that there is a bundle functor $F: \mathcal{M} f_{n} \rightarrow \mathcal{F M}$ with $n \geq k+1$ and a natural operator $A: F \rightsquigarrow Q\left(\operatorname{reg} T_{k}^{r *} \rightarrow K_{k}^{r *}\right)$.

Natural operations with connections have been studied by many authors; see e.g. [2], [4], [5], [7], etc.

From now on $x^{1}, \ldots, x^{n}$ and $t^{1}, \ldots, t^{k}$ are the usual coordinates on $\mathbb{R}^{n}$ and $\mathbb{R}^{k}$ respectively.

All manifolds are assumed to be finite-dimensional and smooth, i.e. of class $\mathcal{C}^{\infty}$. Maps between manifolds are assumed to be smooth.

\section{Torsion free linear connections of order $r$ and the bundle of contact $(k, r)$-elements}

1. The bundle functor $K_{k}^{r}$ of contact $(k, r)$-elements. Let $n$ and $k$ be natural numbers. For every $n$-manifold $M$ we have the bundle $T_{k}^{r} M=$ $J_{0}^{r}\left(\mathbb{R}^{k}, M\right)$ over $M$ of so-called $(k, r)$-velocities on $M$. Every embedding $\varphi$ : $M \rightarrow N$ of $n$-manifolds induces a bundle map $T_{k}^{r} \varphi: T_{k}^{r} M \rightarrow T_{k}^{r} N$ by $T_{k}^{r} \varphi\left(j_{0}^{r} \gamma\right)=j_{0}^{r}(\varphi \circ \gamma)$ for $\gamma: \mathbb{R}^{k} \rightarrow M$. The correspondence $T_{k}^{r}: \mathcal{M} f_{n} \rightarrow$ $\mathcal{F} \mathcal{M}$ is a bundle functor of order $r$ from the category $\mathcal{M} f_{n}$ of $n$-dimensional manifolds and their embeddings into the category $\mathcal{F} \mathcal{M}$ of fibered manifolds and their fibered maps.

Every $\xi=j_{0}^{r} \psi \in L_{k}^{r}=\operatorname{inv} J_{0}^{r}\left(\mathbb{R}^{k}, \mathbb{R}^{k}\right)_{0}$, the Lie group of invertible $r$-jets $\mathbb{R}^{k} \rightarrow \mathbb{R}^{k}$ with source and target $0 \in \mathbb{R}^{k}$, induces a natural automorphism 
$\bar{\xi}: T_{k}^{r} \rightarrow T_{k}^{r}, \bar{\xi}: T_{k}^{r} M \rightarrow T_{k}^{r} M$ by $\bar{\xi}\left(j_{0}^{r} \gamma\right)=j_{0}^{r}\left(\gamma \circ \psi^{-1}\right)$ for $\gamma: \mathbb{R}^{k} \rightarrow M$. This defines a group homomorphism $L_{k}^{r} \rightarrow \operatorname{Aut}\left(T_{k}^{r}\right)$.

It is well known that if $n \geq k+1$ then the above homomorphism is an isomorphism, i.e. $\operatorname{Aut}\left(T_{k}^{r}\right) \cong L_{k}^{r}$.

Assume $n \geq k+1$. For every $n$-manifold $M, \widetilde{T}_{k}^{r} M=\operatorname{reg} J_{0}^{r}\left(\mathbb{R}^{k}, M\right)=$ $\left\{j_{0}^{r} \gamma \mid \gamma: \mathbb{R}^{k} \rightarrow M, \operatorname{rank}\left(d_{0} \gamma\right)=k\right\}$ is an open subbundle of $T_{k}^{r} M$. Elements of $\widetilde{T}_{k}^{r} M$ are called regular $(k, r)$-velocities on $M$. For every embedding $\varphi$ : $M \rightarrow N$ of $n$-manifolds, $T_{k}^{r} \varphi\left(\widetilde{T}_{k}^{r} M\right) \subset \widetilde{T}_{k}^{r} N$, and we let $\widetilde{T}_{k}^{r} \varphi: \widetilde{T}_{k}^{r} M \rightarrow \widetilde{T}_{k}^{r} N$ be the restriction of $T_{k}^{r} \varphi$. The correspondence $\widetilde{T}_{k}^{r}: \mathcal{M} f_{n} \rightarrow \mathcal{F} \mathcal{M}$ is a bundle functor of order $r$.

For every $n$-manifold $M, \widetilde{T}_{k}^{r} M$ is invariant with respect to the action of $L_{k}^{r}=\operatorname{Aut}\left(T_{k}^{r}\right)$ on $T_{k}^{r} M$. So, we have (by restriction) the left action of $L_{k}^{r}$ on $\widetilde{T}_{k}^{r} M$, and the quotient bundle $K_{k}^{r} M=\widetilde{T}_{k}^{r} M / L_{k}^{r}$ over $M$ of socalled contact $(k, r)$-elements. Let $\pi: K_{k}^{r} M \rightarrow M$ be the bundle projection. This bundle was introduced by C. Ehresmann [1]. The quotient projection $\kappa: \widetilde{T}_{k}^{r} M \rightarrow K_{k}^{r} M$ is a principal fiber bundle with structure group $L_{k}^{r}$. The right principal bundle action of $L_{k}^{r}$ on $\widetilde{T}_{k}^{r} M$ is given by $v \cdot \xi=\bar{\xi}^{-1}(v)$ for $\xi \in L_{k}^{r}, v \in \widetilde{T}_{k}^{r} M$. For every embedding $\varphi: M \rightarrow N$ of $n$-manifolds, $\widetilde{T}_{k}^{r} \varphi$ commutes with the left action of $L_{k}^{r}$ on $\widetilde{T}_{k}^{r} M$ and we have the quotient map $K_{k}^{r} \varphi: K_{k}^{r} M \rightarrow K_{k}^{r} N$. Then $\widetilde{T}_{k}^{r} \varphi$ is a principal bundle morphism covering $K_{k}^{r} \varphi$. The correspondence $K_{k}^{r}: \mathcal{M} f_{n} \rightarrow \mathcal{F M}$ is a bundle functor of order $r$.

2. Principal fiber bundle $\kappa^{0}: P^{0} \rightarrow Q^{0}$ and non-existence of $\mathrm{GL}\left(\mathbb{R}^{n}\right)$-invariant connections on $P^{0}$. Let $n \geq k+1$. Let $\kappa^{0}: P^{0} \rightarrow Q^{0}$ be the restriction of $\kappa: \widetilde{T}_{k}^{r} \mathbb{R}^{n} \rightarrow K_{k}^{r} \mathbb{R}^{n}$ to the fibers over $0 \in \mathbb{R}^{n}$. Then $\kappa^{0}$ is a principal fiber bundle (a principal subbundle of $\kappa$ ) with structure group $L_{k}^{r}$. The right action of $L_{k}^{r}$ on $P^{0}$ is the restriction of the right action of $L_{k}^{r}$ on $\widetilde{T}_{k}^{r} \mathbb{R}^{n}$.

There is a left action $\alpha^{0}: \operatorname{GL}\left(\mathbb{R}^{n}\right) \times P^{0} \rightarrow P^{0}$ given by $\alpha^{0}(\eta, p)=\widetilde{T}_{k}^{r} \eta(p)$ for $\eta \in \mathrm{GL}\left(\mathbb{R}^{n}\right), p \in P^{0}$. This action covers the left action $\beta^{0}: \operatorname{GL}\left(\mathbb{R}^{n}\right) \times$ $Q^{0} \rightarrow Q^{0}$ defined by $\beta^{0}(\eta, q)=K_{k}^{r} \eta(q)$ for $\eta \in \mathrm{GL}\left(\mathbb{R}^{n}\right), q \in Q^{0}$. For every $\eta \in \mathrm{GL}\left(\mathbb{R}^{n}\right)$ the mapping $\alpha_{\eta}^{0}=\alpha^{0}(\eta, \cdot): P^{0} \rightarrow P^{0}$ is a principal fiber bundle isomorphism covering $\beta_{\eta}^{0}=\beta^{0}(\eta, \cdot): Q^{0} \rightarrow Q^{0}$.

A connection $\omega: T P^{0} \rightarrow l_{k}^{r}=\operatorname{Lie}\left(L_{k}^{r}\right)$ on $P^{0}$ is called $\mathrm{GL}\left(\mathbb{R}^{n}\right)$-invariant if $\left(\alpha_{\eta}^{0}\right)^{*} \omega=\omega$ for every $\eta \in \mathrm{GL}\left(\mathbb{R}^{n}\right)$.

Proposition 1. There are no $\operatorname{GL}\left(\mathbb{R}^{n}\right)$-invariant connections $\omega: T P^{0}$ $\rightarrow l_{k}^{r}$ on $P^{0}$.

Proof. Suppose $\omega: T P^{0} \rightarrow l_{n}^{r}$ is a $\mathrm{GL}\left(\mathbb{R}^{n}\right)$-invariant connection. According to the general theory of invariant connections (see Kobayashi and 
Nomizu [3]), there is a linear map $\Lambda: \operatorname{gl}\left(\mathbb{R}^{n}\right) \rightarrow l_{k}^{r}$ satisfying the following two conditions:

(1) $\Lambda(X)=\lambda(X)$ for $X \in \operatorname{Lie}(J)$;

(2) $\Lambda(\operatorname{ad}(j)(X))=\operatorname{ad}(\lambda(j)) \Lambda(X)$ for $j \in J$ and $X \in \operatorname{gl}\left(\mathbb{R}^{n}\right)$.

Here $J \subset \mathrm{GL}\left(\mathbb{R}^{n}\right)$ is the stabilizer of $\kappa^{0}\left(\sigma_{0}\right) \in Q^{0}, \sigma_{0}=j_{0}^{r}\left(t^{1}, \ldots, t^{k}, 0, \ldots, 0\right)$ $\in P^{0}$, and $\lambda: \operatorname{Lie}(J) \rightarrow l_{k}^{r}$ is the Lie algebra homomorphism corresponding to the group homomorphism $\lambda: J \rightarrow L_{k}^{r}$, that is, $j . \sigma_{0}=\sigma_{0} \cdot \lambda(j)$ for $j \in J$. We recall that $\Lambda(X)=\omega_{\sigma_{0}}(\widetilde{X})$ for $X \in \operatorname{gl}\left(\mathbb{R}^{n}\right)$, where $\widetilde{X}$ is the vector field on $P^{0}$ induced by $X$.

We have the Lie algebra isomorphism $l_{n}^{r}=J_{0}^{r}\left(T \mathbb{R}^{n}\right)_{0}:=\left\{j_{0}^{r} X \mid X \in\right.$ $\left.\mathcal{X}\left(\mathbb{R}^{n}\right), X_{0}=0\right\}$, where the bracket in $J_{0}^{r}\left(T \mathbb{R}^{n}\right)_{0}$ is given by $\left[j_{0}^{r} X, j_{0}^{r} Y\right]=$ $j_{0}^{r}([Y, X])$ (see $\left.[5]\right)$.

Consider the elements

$$
\begin{aligned}
X^{1} & =j_{0}^{r}\left(x^{1} \frac{\partial}{\partial x^{k+1}}\right), & X^{3} & =j_{0}^{r}\left(x^{k+1} \frac{\partial}{\partial x^{k+1}}\right), \\
X^{2} & =j_{0}^{r}\left(x^{1} \frac{\partial}{\partial x^{1}}\right), & X^{4} & =j_{0}^{r}\left(x^{k+1} \frac{\partial}{\partial x^{1}}\right)
\end{aligned}
$$

of $\operatorname{gl}\left(\mathbb{R}^{n}\right) \subset l_{n}^{r}$. Their one-parameter subgroups in $\mathrm{GL}\left(\mathbb{R}^{n}\right) \subset L_{n}^{r}$ are

$$
\begin{aligned}
& a_{t}^{1}=j_{0}^{r}\left(x^{1}, \ldots, x^{k}, x^{k+1}+t x^{1}, x^{k+2}, \ldots, x^{n}\right), \\
& a_{t}^{2}=j_{0}^{r}\left(e^{t} x^{1}, x^{2}, \ldots, x^{n}\right), \\
& a_{t}^{3}=j_{0}^{r}\left(x^{1}, \ldots, x^{k}, e^{t} x^{k+1}, x^{k+2}, \ldots, x^{n}\right), \\
& a_{t}^{4}=j_{0}^{r}\left(x^{1}+t x^{k+1}, x^{2}, \ldots, x^{n}\right) .
\end{aligned}
$$

Of course $a_{t}^{2}, a_{t}^{3}, a_{t}^{4} \in J, \lambda\left(a_{t}^{2}\right) \neq \mathrm{id}, \lambda\left(a_{t}^{3}\right)=\mathrm{id}, \lambda\left(a_{t}^{4}\right)=$ id and $a_{t}^{1} \in$ $\operatorname{GL}\left(\mathbb{R}^{n}\right) \subset L_{n}^{r}$. Hence $X^{2}, X^{3}, X^{4} \in \operatorname{Lie}(J), X^{1} \in \operatorname{gl}\left(\mathbb{R}^{n}\right), \Lambda\left(X^{3}\right)=\Lambda\left(X^{4}\right)=0$ and $\Lambda\left(X^{2}\right) \neq 0$.

On the other hand if $\varphi=\left(x^{1}+x^{k+1}, x^{2}, \ldots, x^{n}\right)$ then $j=j_{0}^{r} \varphi \in J$ and $\lambda(j)=$ id. So,

$$
\begin{aligned}
\Lambda\left(X^{1}\right) & =\operatorname{ad}(\lambda(j)) \Lambda\left(X^{1}\right)=\Lambda\left(\operatorname{ad}(j) X^{1}\right)=\Lambda\left(j_{0}^{r}\left(\varphi_{*}\left(x^{1} \frac{\partial}{\partial x^{k+1}}\right)\right)\right) \\
& =\Lambda\left(X^{1}+X^{2}-X^{3}-X^{4}\right)=\Lambda\left(X^{1}\right)+\Lambda\left(X^{2}\right),
\end{aligned}
$$

i.e. $\Lambda\left(X^{2}\right)=0$.

This contradiction ends the proof of Proposition 1.

3. Linear connections of order $r$. A linear $r$-order connection on an $n$-manifold $M$ is a vector bundle morphism $\lambda: T M \rightarrow J^{r} T M$ such that $\pi_{0}^{r} \circ \lambda=\operatorname{id}_{T M}$, where $\pi_{0}^{r}: J^{r} T M \rightarrow T M$ is the target projection (see [10]). 
REMARK 1. If $M$ is an $n$-manifold we have the principal fiber bundle $P^{r} M=\operatorname{inv} J_{0}^{r}\left(\mathbb{R}^{n}, M\right)$ over $M$ with standard group $L_{n}^{r}$. The right action of $L_{n}^{r}$ on $P^{r} M$ is given by the composition of jets. If $\varphi: M \rightarrow N$ is an embedding of $n$-manifolds, then we define $P^{r}(\varphi): P(M) \rightarrow P(N)$ by composition of jets. There is a canonical bijection between connections $\omega: T P^{r} M \rightarrow l_{n}^{r}$ on $P^{r} M$ and linear $r$-order connections $\lambda: T M \rightarrow J^{r} T M$ on $M$ by $H^{\omega}=\mathcal{P}^{r} \circ\left(\lambda \times_{M} \operatorname{id}_{P^{r} M}\right)$, where $H^{\omega}: T M \times_{M} P^{r} M \rightarrow T P^{r} M$ is the horizontal lifting morphism of $\omega$, and $\mathcal{P}^{r}: J^{r} T M \times_{M} P^{r} M \rightarrow T P^{r} M$ is the flow morphism of $P^{r}$ (see [10]).

Given an $n$-manifold $M$ we define $C^{r}(M)=\left(\mathrm{id}_{T^{*} M} \otimes \pi_{0}^{r}\right)^{-1}\left(\mathrm{id}_{T M}\right) \subset$ $T^{*} M \otimes J^{r} T M$ to be the subbundle in $T^{*} M \otimes J^{r} T M$, where $\pi_{0}^{r}: J^{r} T M \rightarrow$ $T M$ is the target projection. It is called the bundle of linear $r$-order connections on $M$. The sections of $C^{r}(M)$ are exactly the linear $r$-order connections on $M$. For every embedding $\varphi: M \rightarrow N$ the mapping $T^{*} \varphi \otimes J^{r} T \varphi$ : $T^{*} M \otimes J^{r} T M \rightarrow T^{*} N \otimes J^{r} T N$ sends $C^{r}(M)$ into $C^{r}(N)$ and we have (by restriction) a fiber bundle map $C^{r}(\varphi): C^{r}(M) \rightarrow C^{r}(N)$. The correspondence $C^{r}: \mathcal{M} f_{n} \rightarrow \mathcal{F M}$ is a bundle functor of order $r+1$.

An $r$-order linear connection $\lambda: T M \rightarrow J^{r} T M$ is called torsion free if $\{\lambda(u), \lambda(v)\}=0$ for any $u, v \in T_{x} M, x \in M$. Here $\{\cdot, \cdot\}: J^{r} T M \times_{M}$ $J^{r} T M \rightarrow J^{r-1} T M$ is the algebraic bracket given by

$$
\left\{j_{x}^{r} X, j_{x}^{r} Y\right\}=j_{x}^{r-1}([X, Y])
$$

(see $[10])$.

By the Frobenius theorem, an $r$-order connection $\lambda$ on an $n$-manifold $M$ is torsion free iff for each $x \in M$ there is a chart $\Phi$ near $x$ such that $\Phi(x)=0$ and $C^{r}(\Phi)\left(\lambda_{x}\right)=\lambda^{0}$, where $\lambda^{0}: T_{0} \mathbb{R}^{n} \rightarrow J_{0}^{r} T \mathbb{R}^{n}$ is defined by $\lambda^{0}(u)=j_{0}^{r} \widetilde{u}$ for $u \in T_{0} \mathbb{R}^{n}$ and $\widetilde{u}$ is the constant vector field on $\mathbb{R}^{n}$ such that $\widetilde{u}_{0}=u$. The coordinates $\Phi$ corresponding to $\Phi$ are called normal coordinates of $\lambda$ at $x$ (see [10]). Clearly, if $\bar{\Phi}$ are another normal coordinates of $\lambda$ at $x$ then $\bar{\Phi}=A \circ \Phi$ for some $A \in \mathrm{GL}\left(\mathbb{R}^{n}\right)$.

Given an $n$-manifold $M$ we define $C_{0}^{r}(M)$ to be the orbit in $T^{*} M \otimes J^{r} T M$ of $\lambda^{0}$ with respect to embeddings $\psi: \mathbb{R}^{n} \rightarrow M$, where $\lambda^{0} \in\left(T_{0}^{*} \mathbb{R}^{n}\right) \otimes$ $\left(J_{0}^{r} T \mathbb{R}^{n}\right)$ is as above. It is a subbundle of $T^{*} M \otimes J^{r} T M$ and it is called the bundle of torsion free linear $r$-order connections on $M$. The sections of $C_{0}^{r}(M)$ are exactly the torsion free linear $r$-order connections on $M$. For every embedding $\varphi: M \rightarrow N$ the mapping $T^{*} \varphi \otimes J^{r} T \varphi: T^{*} M \otimes J^{r} T M \rightarrow$ $T^{*} N \otimes J^{r} T N$ sends $C_{0}^{r}(M)$ into $C_{0}^{r}(N)$ and we have (by restriction) a fiber bundle map $C_{0}^{r}(\varphi): C_{0}^{r}(M) \rightarrow C_{0}^{r}(N)$. The correspondence $C_{0}^{r}: \mathcal{M} f_{n} \rightarrow$ $\mathcal{F} \mathcal{M}$ is a bundle functor of order $r+1$.

4. Non-existence of natural operators $C_{0}^{r} \rightsquigarrow Q\left(\widetilde{T}_{k}^{r} \rightarrow K_{k}^{r}\right)$. Let $P \rightarrow \underline{P}$ be a principal fiber bundle with structure group $L_{k}^{r}$ and $m$-dimensio- 
nal base. According to the general theory, connections $T P \rightarrow l_{k}^{r}$ on $P$ can be identified with sections of some fiber bundle $Q P=Q(P \rightarrow \underline{P})$ over $\underline{P}$, called the bundle of connections on $P$. It is known that every local principal bundle isomorphism $\Psi:(P \rightarrow \underline{P}) \rightarrow(H \rightarrow \underline{H})$ covering $\underline{\Psi}: \underline{P} \rightarrow \underline{H}$ induces functorially a fiber bundle map $Q \Psi: Q P \rightarrow Q H$ over $\underline{\Psi}$. The correspondence $Q: \mathcal{P}_{m}\left(L_{k}^{r}\right) \rightarrow \mathcal{F} \mathcal{M}$ is a gauge bundle functor from the category $\mathcal{P}_{m}\left(L_{k}^{r}\right)$ of principal fiber bundles with structure group $L_{k}^{r}$ and $m$-dimensional bases and their local principal bundle isomorphisms (see [5]).

According to [5] a natural operator $A: C_{0}^{r} \rightsquigarrow Q\left(\widetilde{T}_{k}^{r} \rightarrow K_{k}^{r}\right)$ over $n$ manifolds is a family of regular operators

$$
A: C_{M}^{\infty}\left(C_{0}^{r}(M)\right) \rightarrow C_{K_{k}^{r} M}^{\infty}\left(Q\left(\widetilde{T}_{k}^{r} M \rightarrow K_{k}^{r} M\right)\right)
$$

from the set of torsion free $r$-order linear connections on $M$ (sections of $\left.C_{0}^{r}(M)\right)$ into the set of connections on $\kappa: \widetilde{T}_{k}^{r} M \rightarrow K_{k}^{r} M$ (sections of $\left.Q\left(\widetilde{T}_{k}^{r} M \rightarrow K_{k}^{r} M\right)\right)$ for every $n$-manifold $M$ such that the naturality condition with respect to $\mathcal{M} f_{n}$-morphisms is satisfied. This means that for every $\lambda \in C_{M}^{\infty}\left(C_{0}^{r}(M)\right)$ and $\bar{\lambda} \in C_{N}^{\infty}\left(C_{0}^{r}(N)\right)$ and every $\mathcal{M} f_{n}$-morphism $\varphi: M \rightarrow N$, if $\bar{\lambda}$ and $\lambda$ are $\varphi$-related, then $A(\bar{\lambda})$ and $A(\lambda)$ are $Q \widetilde{T}_{k}^{r} \varphi$-related.

The first main result of this paper is the following theorem.

TheOREM 1. Let $n, k$ and $r$ be natural numbers such that $n \geq k+1$. There are no natural operators $C_{0}^{r} \rightsquigarrow Q\left(\widetilde{T}_{k}^{r} \rightarrow K_{k}^{r}\right)$ over $n$-manifolds.

Proof. Suppose that $A: C_{0}^{r} \rightsquigarrow Q\left(\widetilde{T}_{k}^{r} \rightarrow K_{k}^{r}\right)$ is a natural operator over $n$-manifolds. We define $H^{\omega}: T Q^{0} \times{ }_{Q^{0}} P^{0} \rightarrow T P^{0}$ by

$$
H^{\omega}(u, v)=H^{A\left(\widetilde{\lambda}^{0}\right)}(u, v)
$$

for $u \in T_{\sigma} Q^{0}, \sigma \in Q^{0}, v \in P^{0}, \kappa^{0}(v)=\sigma$, where $H^{A\left(\widetilde{\lambda}^{0}\right)}: T K_{k}^{r} \mathbb{R}^{n} \times K_{k}^{r} \mathbb{R}^{n}$ $\widetilde{T}_{k}^{r} \mathbb{R}^{n} \rightarrow T \widetilde{T}_{k}^{r} \mathbb{R}^{n}$ is the horizontal lifting morphism of $A\left(\widetilde{\lambda}^{0}\right)$ and where $\widetilde{\lambda}^{0}$ is the translation invariant section of $C_{0}^{r}\left(\mathbb{R}^{n}\right)$ such that $\widetilde{\lambda}_{\mid 0}^{0}=\lambda^{0}$. Here $\lambda_{0}$ is the element from $C_{0}^{r}\left(\mathbb{R}^{n}\right)$ as in Section 3 .

Clearly, $H^{\omega}(u, v) \in T_{v} \widetilde{T}_{k}^{r} \mathbb{R}^{n}$ and it projects on $u$ under $T \kappa$. Hence $H^{\omega}(u, v)$ is $\left(\widetilde{T}_{k}^{r} \mathbb{R}^{n} \rightarrow \mathbb{R}^{n}\right)$-vertical because $u$ is $\pi$-vertical. Therefore, $H^{\omega}(u, v)$ $\in T P^{0}$. Since $H^{\Lambda\left(\widetilde{\lambda}^{0}\right)}$ is a horizontal lifting, so is $H^{\omega}$.

Let $\omega: T P^{0} \rightarrow l_{k}^{r}$ be the corresponding connection on $P^{0}$. Since $\widetilde{\lambda}^{0}$ is $\operatorname{GL}\left(\mathbb{R}^{n}\right)$-invariant, so is $\Lambda\left(\widetilde{\lambda}^{0}\right)$ because of the naturality od $\Lambda$. Hence $\omega$ is $\mathrm{GL}\left(\mathbb{R}^{n}\right)$-invariant.

This is a contradiction by Proposition 1 .

We have the following obvious corollaries of Theorem 1.

COROLlary 1 . Let $n, k$ and $r$ be natural numbers such that $n \geq k+1$. There are no natural operators $C^{r} \rightsquigarrow Q\left(\widetilde{T}_{k}^{r} \rightarrow K_{k}^{r}\right)$ over $n$-manifolds. 
Corollary 2. Let $n, k$ and $r$ be natural numbers such that $n \geq k+1$. There are no canonical connections $\omega: T \widetilde{T}_{k}^{r} M \rightarrow l_{k}^{r}$ on $T \widetilde{T}_{k}^{r} M$ over $n$ manifolds.

5. A generalization. Using the same method as in the proof of Theorem 1 we obtain the following general fact.

THEOREM 2. Let $n, k$ and $r$ be natural numbers such that $n \geq k+1$. Let $F: \mathcal{M} f_{n} \rightarrow \mathcal{F M}$ be a bundle functor such that there is a $\mathrm{GL}\left(\mathbb{R}^{n}\right)$-invariant element $\mu^{0} \in F_{0} \mathbb{R}^{n}$. There are no natural operators $A: F \rightsquigarrow Q\left(\widetilde{T}_{k}^{r} \rightarrow K_{k}^{r}\right)$.

Proof. In the proof of Theorem 1 we replace $\widetilde{\lambda}^{0}$ by the translation invariant section $\widetilde{\mu}^{0}$ of $F \mathbb{R}^{n}$ such that $\widetilde{\mu}_{\mid 0}^{0}=\mu^{0}$.

REMARK 2 . There are many natural bundles $F M$ satisfying the assumption of Theorem 2. For example, $C_{0}^{s}(M)$ and $C^{s}(M)$ for all $s$, fiber products $C_{0}^{s_{1}}(M) \times{ }_{M} \ldots \times_{M} C_{0}^{s_{K}}(M), T^{A} M$ for all Weil algebras $A$, all natural vector bundles $\left(\bigotimes^{p} T^{*} M \otimes \bigotimes^{q} T M, T_{k}^{r} M, T_{k}^{r *} M, \ldots\right)$, etc.

COROLlary 3. Let $n, k$ and $r$ be natural numbers such that $n \geq k+1$. There are no natural operators $\Lambda$ sending a generalized connection $\Gamma$ on $\widetilde{T}_{k}^{r} M \rightarrow M\left(\right.$ or $\left.K_{k}^{r} M \rightarrow M\right)$ and a linear connection $\nabla$ on $M$ to a connection $\Lambda(\Gamma, \nabla): T \widetilde{T}_{k}^{r} M \rightarrow l_{k}^{r}$.

Proof. Every $r$-order linear connection $\lambda: T M \rightarrow J^{r} T M$ on $M$ gives rise to a generalized connection $\Gamma$ on $\widetilde{T}_{k}^{r} M \rightarrow M$ (or $K_{k}^{r} M \rightarrow M$ ) because $\widetilde{T}_{k}^{r}\left(\right.$ or $\left.K_{k}^{r}\right)$ is of order $r$. Next we apply Theorem 2 and Remark 2 .

The following example shows that Theorem 1 is not true for an arbitrary natural bundle $F$ instead of $C_{0}^{r}$.

EXAMPLE 1. Let $F=P^{r+1}: \mathcal{M} f_{n} \rightarrow \mathcal{F M}$ be the bundle functor from Remark 1. Consider a connection $\omega: T \widetilde{T}_{k}^{r} \mathbb{R}^{n} \rightarrow l_{k}^{r}$ on $\kappa: \widetilde{T}_{k}^{r} \mathbb{R}^{n} \rightarrow$ $K_{k}^{r} \mathbb{R}^{n}$. Given a section $\varrho$ of $F M$ define $A(\varrho): T \widetilde{T}_{k}^{r} M \rightarrow l_{k}^{r}$ by $A(\varrho)(v)=$ $\omega\left(T \widetilde{T}_{k}^{r} \varphi^{-1}(v)\right)$ for $j_{0}^{r+1} \varphi=\varrho(x), v \in\left(T \widetilde{T}_{k}^{r} M\right)_{x}, x \in M$. Then $A(\varrho)$ is a connection on $\kappa: \widetilde{T}_{k}^{r} M \rightarrow K_{k}^{r} M$. In this way we obtain a natural operator $A: F \rightsquigarrow Q\left(\widetilde{T}_{k}^{r} \rightarrow K_{k}^{r}\right)$.

\section{Torsion free linear connections of order $r$ and the bundle functor of contact $(k, r)$-coelements}

6. The bundle functor $K_{k}^{r *}$ of contact $(k, r)$-coelements. Let $n$ and $k$ be natural numbers. For every $n$-manifold $M$ we have the bundle $T_{k}^{r *} M=$ $J^{r}\left(M, \mathbb{R}^{k}\right)_{0}$ over $M$ of so-called $(k, r)$-covelocities on $M$. Every embedding $\varphi: M \rightarrow N$ of $n$-manifolds induces a bundle map $T_{k}^{r *} \varphi: T_{k}^{r *} M \rightarrow T_{k}^{r *} N$ 
by $T_{k}^{r *} \varphi\left(j_{x}^{r} \gamma\right)=j_{\varphi(x)}^{r}\left(\gamma \circ \varphi^{-1}\right)$ for $\gamma: M \rightarrow \mathbb{R}^{k}, x \in M, \gamma(x)=0$. The correspondence $T_{k}^{r *}: \mathcal{M} f_{n} \rightarrow \mathcal{F M}$ is a bundle functor of order $r$.

Every $\xi=j_{0}^{r} \psi \in L_{k}^{r}$ induces a natural automorphism $\bar{\xi}: T_{k}^{r *} \rightarrow T_{k}^{r *}$, $\bar{\xi}: T_{k}^{r *} M \rightarrow T_{k}^{r *} M$, defined by $\bar{\xi}\left(j_{x}^{r} \gamma\right)=j_{x}^{r}(\psi \circ \gamma)$ for $\gamma: M \rightarrow \mathbb{R}^{k}, x \in M$, $\gamma(x)=0$. This defines a group homomorphism $L_{k}^{r} \rightarrow \operatorname{Aut}\left(T_{k}^{r *}\right)$

If $n \geq k+1$ then the above homomorphism is an isomorphism, i.e. $\operatorname{Aut}\left(T_{k}^{r *}\right) \cong L_{k}^{r}$.

Assume $n \geq k+1$. For every $n$-manifold $M, \widetilde{T}_{k}^{r *} M=\operatorname{reg} J^{r}\left(M, \mathbb{R}^{k}\right)_{0}=$ $\left\{j_{x}^{r} \gamma \mid \gamma: M \rightarrow \mathbb{R}^{k}, x \in M, \gamma(x)=0, \operatorname{rank}\left(d_{x} \gamma\right)=k\right\}$ is an open subbundle of $T_{k}^{r *} M$. Elements of $\widetilde{T}_{k}^{r *} M$ are called regular $(k, r)$-covelocities on $M$. For every embedding $\varphi: M \rightarrow N$ of $n$-manifolds, $T_{k}^{r *} \varphi\left(\widetilde{T}_{k}^{r *} M\right) \subset \widetilde{T}_{k}^{r *} N$, and we let $\widetilde{T}_{k}^{r *} \varphi: \widetilde{T}_{k}^{r *} M \rightarrow \widetilde{T}_{k}^{r *} N$ be the restriction of $T_{k}^{r *} \varphi$. The correspondence $\widetilde{T}_{k}^{r *}: \mathcal{M} f_{n} \rightarrow \mathcal{F M}$ is a bundle functor of order $r$.

For every $n$-manifold $M, \widetilde{T}_{k}^{r *} M$ is invariant with respect to the action of $L_{k}^{r}=\operatorname{Aut}\left(T_{k}^{r *}\right)$ on $T_{k}^{r *} M$. So, we have (by restriction) the left action of $L_{k}^{r}$ on $\widetilde{T}_{k}^{r *} M$, and the quotient bundle $K_{k}^{r *} M=\widetilde{T}_{k}^{r *} M / L_{k}^{r}$ over $M$ of so called contact $(k, r)$-coelements. This bundle was introduced in [9]. The quotient projection $\kappa^{*}: \widetilde{T}_{k}^{r *} M \rightarrow K_{k}^{r *} M$ is a principal fiber bundle with structure group $L_{k}^{r}$. The right principal bundle action of $L_{k}^{r}$ on $\widetilde{T}_{k}^{r *} M$ is given by $v . \xi=\bar{\xi}^{-1}(v)$ for $\xi \in L_{k}^{r}, v \in \widetilde{T}_{k}^{r *} M$. For every embedding $\varphi: M \rightarrow N$ of $n$-manifolds, $\widetilde{T}_{k}^{r *} \varphi$ commutes with the left action of $L_{k}^{r}$ on $\widetilde{T}_{k}^{r *} M$ and we have the quotient map $K_{k}^{r *} \varphi: K_{k}^{r *} M \rightarrow K_{k}^{r *} N$. Then $\widetilde{T}_{k}^{r *} \varphi$ is a principal bundle morphism covering $K_{k}^{r *} \varphi$. The correspondence $K_{k}^{r *}: \mathcal{M} f_{n} \rightarrow \mathcal{F} \mathcal{M}$ is a bundle functor of order $r$ (see [9]).

7. Principal fiber bundle $\kappa^{* 0}: P^{* 0} \rightarrow Q^{* 0}$ and non-existence of $\operatorname{GL}\left(\mathbb{R}^{n}\right)$-invariant connections on $P^{* 0}$. Let $n \geq k+1$. Let $\kappa^{* 0}: P^{* 0} \rightarrow$ $Q^{* 0}$ be the restriction of $\kappa^{*}: \widetilde{T}_{k}^{r *} \mathbb{R}^{n} \rightarrow K_{k}^{r *} \mathbb{R}^{n}$ to the fibers over $0 \in \mathbb{R}^{n}$. Then $\kappa^{* 0}$ is a principal fiber bundle (a principal subbundle of $\kappa^{*}$ ) with structure group $L_{k}^{r}$. Its right action of $L_{k}^{r}$ on $P^{* 0}$ is the restriction of the right action of $L_{k}^{r}$ on $\widetilde{T}_{k}^{r *} \mathbb{R}^{n}$.

There is a left action $\alpha^{* 0}: \operatorname{GL}\left(\mathbb{R}^{n}\right) \times P^{* 0} \rightarrow P^{* 0}$ defined by $\alpha^{* 0}(\eta, p)=$ $\widetilde{T}_{k}^{r *} \eta(p)$ for $\eta \in \mathrm{GL}\left(\mathbb{R}^{n}\right), p \in P^{* 0}$. This action covers the left action $\beta^{* 0}$ : $\mathrm{GL}\left(\mathbb{R}^{n}\right) \times Q^{* 0} \rightarrow Q^{* 0}$ given by $\beta^{* 0}(\eta, q)=K_{k}^{r *} \eta(q)$ for $\eta \in \mathrm{GL}\left(\mathbb{R}^{n}\right), q \in Q^{* 0}$. For every $\eta \in \operatorname{GL}\left(\mathbb{R}^{n}\right)$ the mapping $\alpha_{\eta}^{* 0}=\alpha^{* 0}(\eta, \cdot): P^{* 0} \rightarrow P^{* 0}$ is a principal fiber bundle isomorphism covering $\beta_{\eta}^{* 0}=\beta^{* 0}(\eta, \cdot): Q^{* 0} \rightarrow Q^{* 0}$.

A connection $\omega: T P^{* 0} \rightarrow l_{k}^{r}=\mathcal{L}\left(L_{k}^{r}\right)$ on $P^{* 0}$ is called $\mathrm{GL}\left(\mathbb{R}^{n}\right)$-invariant if $\left(\alpha_{\eta}^{* 0}\right)^{*} \omega=\omega$ for every $\eta \in \mathrm{GL}\left(\mathbb{R}^{n}\right)$.

Proposition 2. There are no $\mathrm{GL}\left(\mathbb{R}^{n}\right)$-invariant connections $\omega: T P^{* 0}$ $\rightarrow l_{k}^{r}$ on $P^{* 0}$. 
Proof. Similar to the proof of Proposition 1.

8. Non-existence of natural operators $C_{0}^{r} \rightsquigarrow Q\left(\widetilde{T}_{k}^{r *} \rightarrow K_{k}^{r}\right)$. The definition of natural operators $C_{0}^{r} \rightsquigarrow Q\left(\widetilde{T}_{k}^{r *} \rightarrow K_{k}^{r *}\right)$ is similar to that of natural operators $C_{0}^{r} \rightsquigarrow Q\left(\widetilde{T}_{k}^{r} \rightarrow K_{k}^{r}\right)$.

The second main result of this paper is the following theorem.

THEOREM 3. Let $n, k$ and $r$ be natural numbers such that $n \geq k+1$. There are no natural operators $C_{0}^{r} \rightsquigarrow Q\left(\widetilde{T}_{k}^{r *} \rightarrow K_{k}^{r *}\right)$ over $n$-manifolds.

Proof. The proof is similar to that of Theorem 1. One uses Proposition 2 instead of Proposition 1.

We have the following obvious corollaries of Theorem 3 .

Corollary 4. Let $n, k$ and $r$ be natural numbers such that $n \geq k+1$. There are no natural operators $C^{r} \rightsquigarrow Q\left(\widetilde{T}_{k}^{r *} \rightarrow K_{k}^{r *}\right)$ over $n$-manifolds.

COROLlary 5. Let $n, k$ and $r$ be natural numbers such that $n \geq k+1$. There are no canonical connections $\omega: T \widetilde{T}_{k}^{r *} M \rightarrow l_{k}^{r}$ on $\widetilde{T}_{k}^{r *} M$ over $n$ manifolds.

9. A generalization. Using the same method as in the proof of Theorem 2 we obtain the following general fact.

TheOREM 4. Let $n, r, k$ be natural numbers such that $n \geq k+1$. Let $F: \mathcal{M} f_{n} \rightarrow \mathcal{F} \mathcal{M}$ be a bundle functor such that there is a $\mathrm{GL}\left(\mathbb{R}^{n}\right)$-invariant element $\mu^{0} \in F_{0} \mathbb{R}^{n}$. Then there are no natural operators $A: F \rightsquigarrow$ $Q\left(\widetilde{T}_{k}^{r *} \rightarrow K_{k}^{r *}\right)$.

COROLlary 6 . Let $n, r, k$ be natural numbers such that $n \geq k+1$. There are no natural operators $\Lambda$ sending a generalized connection $\Gamma$ on $\widetilde{T}_{k}^{r *} M \rightarrow$ $M\left(\right.$ or $\left.K_{k}^{r *} M \rightarrow M\right)$ and a linear connection $\nabla$ on $M$ to a connection $\Lambda(\Gamma, \nabla): T \widetilde{T}_{k}^{r *} M \rightarrow l_{k}^{r}$.

Remark 3. Modifying Example 1 we can show that Theorem 3 is not true for an arbitrary natural bundle $F$ instead of $C_{0}^{r}$.

\section{References}

[1] C. Ehresmann, Introduction à la théorie des structures infinitésimales et des pseudogroupes de Lie, in: Colloque de topologie et géométrie différentielle (Strasbourg, 1952), 97-110.

[2] J. Gancarzewicz and I. Koláŕ, Some gauge natural operators on linear connections, Monatsh. Math. 111 (1991), 23-33.

[3] S. Kobayashi and K. Nomizu, Foundations of Differential Geometry, Interscience, New York, 1963. 
[4] I. Koláŕ, Gauge natural operators transforming connections to the tangent bundle, in: The Mathematical Heritage of C. F. Gauss, World Sci., Singapore, 1991, 416-426.

[5] I. Koláŕ, P. W. Michor and J. Slovák, Natural Operations in Differential Geometry, Springer, 1993.

[6] I. Koláŕ and W. M. Mikulski, Contact elements on fibered manifolds, Czechoslovak Math. J., to appear.

[7] - - - Natural lifting of connections to vertical bundles, Rend. Circ. Mat. Palermo Suppl. 63 (2000), 97-102.

[8] M. Kureš and W. M. Mikulski, Natural lifting of vector fields to bundles of Weil contact elements, Czechoslovak Math. J., to appear.

[9] W. M. Mikulski, On the contact $(k, r)$-coelements, Demonstratio Math., to appear.

[10] M. Paluszny and A. Zajtz, Foundation of the Geometry of Natural Bundles, Lecture Notes, Univ. Caracas, 1984.

Institute of Mathematics

Jagiellonian University

Reymonta 4

30-059 Kraków, Poland

E-mail: mikulski@im.uj.edu.pl 